\title{
Contribution à la connaissance des cours d'eaux méditerranéens de basse altitude : le réseau hydrographique de l'Arc (Bouches-du- Rhône, France). Etat des peuplements en référence aux perturbations anthropiques
}
J.J. Musso'
G. Prévot 1
P. Légier ${ }^{1}$
C. Playoust ${ }^{1}$

Mots clés : Hydrosystème méditerranéen perturbé, invertébrés benthiques, typologie, hydrochimie.

Le réseau hydrographique de la rivière Arc s'étend dans une région fortement urbanisée et industrialisée où l'agriculture joue un rôle encore important. Ce travail présente un compte rendu global sur la situation chimique des eaux et sur les peuplements de la rivière, réalisé au cours d'un cycle annuel à partir d'analyses multivariées (18 paramètres physicochimiques, 17 stations d'étude : 9 sur le cours principal, 8 sur les affluents). Les situations hydrochimiques ainsi mises en évidence sont ordonnées selon un gradient d'eutrophie représenté par la première composante principale. Les éléments allogènes, qui jouent un rôle prépondérant dans le fonctionnement de cet hydrosystème, déterminent une eutrophication qui masque l'eutrophisation naturelle de la rivière. Seuls quelques affluents exempts de pollution sont encore proches de leur situation originelle.

Les peuplements benthiques (151 taxons) du réseau hydrographique de l'Arc (15 points d'échantillonnage pris sur le cours d'eau axial et les affluents) ont été étudiés au moyen d'une analyse factorielle des correspondances. Les stations du cours supérieur se caractérisent par la présence d'espèces lénitophiles adaptées au régime temporaire des milieux aquatiques. Dans la majeure partie du cours principal, la forte pollution organique et chimique est favorable à la prolifération d'espèces saprobiontes. Les affluents, peu ou pas perturbés, hébergent un peuplement se rattachant globalement au biocénotype du rhithron et constituent un réservoir d'espèces susceptibles de recoloniser le cours axial de la rivière.

Contribution to the knowledge of low altitude mediterranean rivers : the hydrographic system of the River Arc (Bouchesdu-Rhône, France). Benthic invertebrates overview

Keywords : Disturbed mediterranean hydrosystem, benthic invertebrates, typology, hydrochemical activity.

The Arc river's hydrographic system is situated in a heavily urbanised and industrialized area where agriculture nevertheless still plays an important role. This paper presents an overall view of the water quality and the benthic populations based on a multivariate analysis of an annual cycle (18 physico-chemical parameters, 17 study sites : 9 on the river's main course, 8 on its tributaries). The resultant hydrochemical states are arranged in order of an eutrophication gradient represented by the first principal component. The allogenic elements, which have a preponderant role in the hydrosystem's activity, cause man-made eutrophication which overshadows the river's natural eutrophication. Only a few unpolluted tributaries still remain close to their original state.

The benthic populations of the Arc hydrographic network were studied through a correspondence analysis using 15 sampling points containing 151 taxa selected on the axial stream and its tributaries. The upstrean stations can be characterized by the presence of lenitophilous species adapted to the transitory environment. Throughout most of the river's course, a high level of organic and chemical pollution has helped to proliferate saprobiont species. In the little or undisturbed tributaries, there is a population which can be basically categorized as a rhithron biocenotype containing species capable of recolonizing the course of the main river.

1. Laboratoire d'Ecologie, Université d'Aix-Marseille III, Avenue Escadrille Normandie-Niemen (case 331), 13397 Marseille Cedex 13. 


\section{Introduction}

L'étude hydrobiologique des rivières et fleuves du Sud-Est de la France est une entreprise récente, c'est pourquoi les données concernant leurs structure et fonctionnement sont encore rares et éparses. Ce sont principalement les travaux de Dia (1978) et GalvinChabrière (1982) sur l'Argens, de Bouzidi (1983) sur l'Aille, de R. Prévot (1984) sur la Moyenne Durance, de Vespini (1985) sur le Buëch et de Galvin (1989) sur le Verdon.

L'Arc, principale rivière du département des Bouches-du-Rhône, n'a été jusqu'à ce jour étudiée que de façon très ponctuelle. Ce cours d'eau subit depuis la source jusqu'à l'embouchure des nuisances et des agressions de toutes sortes, préjudiciables à l'équilibre des biocénoses animales et végétales. Son réseau hydrographique occupe en effet une région fortement urbanisée, très industrialisée où l'agriculture joue un rôle non négligeable.

\section{Le milieu et choix des stations}

L'Arc est un fleuve côtier méditerranéen du SudEst de la France dont le bassin versant $\left(780 \mathrm{~km}^{2}\right)$ est situé en Provence occidentale calcaire (fig. 1). Cette rivière prend sa source à $467 \mathrm{~m}$ d'altitude et se jette, après un parcours est-ouest de $85 \mathrm{~km}$, dans la partie septentrionale de l'étang de Berre.

Le climat de cette région est typiquement méditerranéen (précipitations: moyenne annuelle de $638 \mathrm{~mm}$ sur 18 ans ; températures moyennes extrêmes : $4^{\circ} \mathrm{C}$ en janvier $1985 ; 28,1^{\circ} \mathrm{C}$ en juillet avec une sécheresse estivale très marquée).

D'un point de vue hydrologique, le réseau hydrographique de l'Arc comprend quelques sources à faible débit, 10 affluents de plus ou moins grande importance et plusieurs étendues d'eau artificielles. La pente de l'Arc présente une faible dénivelée $(0,54 \%$ en moyenne) ; son régime est de type méditerranéen pluvial à étiage très bas et crues très violentes. L'importance du rôle du débit dans le fonctionnement de ces écosystèmes, corrélativement à l'évolution des composantes chimiques de l'eau, a fréquemment été soulignée par les auteurs (Lavandier \& Mur 1974).

L'Arc est l'exutoire principal des affluents issus des activités humaines dans la partie Est du département des Bouches-du-Rhône. Les principaux foyers de pollution, localisés sur la figure 1, peuvent être rattachés à 3 secteurs d'activités : urbanisation, activités industrielles, agriculture et élevage porcin.

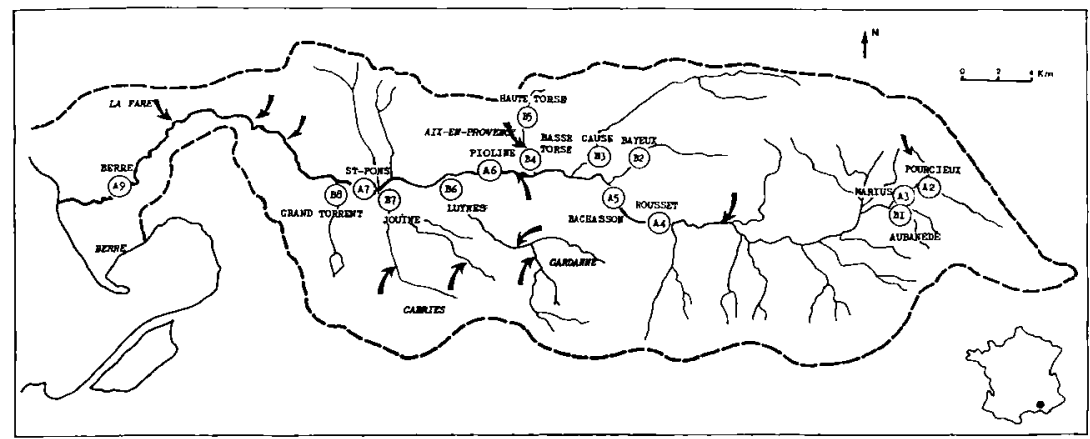

Fig. 1. Bassin hydrographique de l'Arc.

Localisation des stations (cercles) et des impacts (flèches) (les stations prospectées dans le cours principal de l'Arc sont désignées par la lettre A ; celles prises sur les affluents par la lettre B). 
Deux types de stations d'études ont été retenus (fig. 1) :

- sur le cours principal, neuf stations (Al à A9) afin d'établir un diagnostic écologique des eaux de ce fleuve.

- sur les affluents, huit stations (B1 à B8) sur sept des affluents prospectés. Chacun d'eux présente une situation écologique particulière liée à l'action ou à l'absence d'agents polluants d'origines diverses.

Chimie et fonctionnement hydrochimique.

La chimie des eaux de l'Arc a été étudiée dans 17 stations sur 18 paramètres. Ce travail a été réalisé au cours de 11 séries de mesures (tous les 2 mois) s'échelonnant entre novembre 1983 et juillet 1985 (Tableau I)

Une analyse en composantes principales exécutée à partir des 18 paramètres sur un cycle annuel a permis d'établir la courbe d'évolution de la moyenne annuelle des coordonnées stationnelles sur l'axe I de l'A.C.P. en fonction de leur distance par rapport à la source (fig. 2). Elle montre que :

a) dans le cours principal, les stations A1, A2, A3 et A4 se succèdent selon une séquence géographique naturelle de la source vers l'épirhithral ; la chimie des eaux de la haute vallée de l'Arc subit un enrichissement progressif en sels nutritifs, plus marqué en A4. La station A5 est moins chargée en éléments polluants que la station A4 située plus en amont.
Cette dernière subit en effet un apport contaminant important (proximité d'élevages porcins). La trajectoire de la courbe qui prend en compte les coordonnées des 4 stations du cours inférieur (A6, A7, A8, A9) s'inverse : la station A6 (en aval des rejets de la station d'épuration d'Aix-en-Provence) possède les coordonnées les plus fortement négatives sur l'axe ; la station A9 est, dans ce tronçon de l'Arc, la moins chargée en éléments polluants.

b) dans les affluents, à l'exception de la Luyne (B6), les affluents sont en général moins pollués que le cours de l'Arc au niveau de leurs confluences respectives (position positive sur la première composante principale). La contribution des affluents à l'évolution de l'hydrochimisme du cours axial n'est pas évidente ; cette absence de relation tend à montrer la prépondérance des apports eutrophisants directs sur le fonctionnement intrinsèque du cours axial.

L'information générale apportée par cette A.C.P. fait donc ressortir la dominance et l'importance des apports allogènes dans le fonctionnement de l'hydrosystème Arc. Cet enrichissement provenant des nombreuses activités humaines du bassin versant est défini par Duvignaud (1974) sous le terme d'eutrophication. Un phénomène identique est décrit dans les synthèses hydrochimiques sur le Lot (Angelier et al. 1978), l'Ardèche (Doledec 1985) et le Buëch (Vespini et al. 1988).

Tableau I. Caractéristiques physico-chimiques des eaux du bassin de l'Arc dans les stations étudiées (moyenne des mesures effectuées tous les 2 mois, de novembre 1983 à juillet 1985). Stations sur le cours principal : A1 à A9 ; sur les affluents : B1 à B8.

\begin{tabular}{|c|c|c|c|c|c|c|c|c|c|c|c|c|c|c|c|c|c|c|}
\hline & $\begin{array}{l}m_{2} \\
=/ 1\end{array}$ & $\begin{array}{l}\mathrm{NO}_{3} \\
\mathrm{~W}_{5} / 1\end{array}$ & $\begin{array}{l}m n_{i}^{*} \\
m / 1\end{array}$ & $\begin{array}{l}\mathrm{HCO}_{3} \\
-8 / 1\end{array}$ & $\begin{array}{l}\text { Pol- } \\
\operatorname{mg} / 2\end{array}$ & $\begin{array}{l}\mathrm{Cs}^{4+} \\
=\mathrm{k}\end{array}$ & $m_{n}^{* *} / 1$ & $\begin{array}{l}\mathrm{Cl}^{\circ} \\
\mathrm{m} / 1\end{array}$ & $\begin{array}{l}\text { H.E.s.o } \\
\mathrm{mg}_{\mathrm{g}} / \mathrm{l}\end{array}$ & $\begin{array}{c}\text { M.z.s.e } \\
=\pi / 1\end{array}$ & $\begin{array}{l}\text { n.0.ch } \\
\mathrm{Mg}_{\mathrm{g}} \cdot 0_{2} / 1\end{array}$ & $\begin{array}{l}\text { H.O.tr } \\
=g .0_{2} / 1\end{array}$ & $\begin{array}{l}0.0 .0 .5 \\
\mathrm{MR}_{2} .02 / 1\end{array}$ & $\mathrm{pH}$ & $T^{*}$ & $\mathrm{O}_{2}$ & $\begin{array}{c}c \\
\mu 5 / \mathrm{cm}\end{array}$ & $\begin{array}{l}50_{4}^{--} \\
=\mathrm{g} / 1\end{array}$ \\
\hline$A_{1}$ & 0,06 & 0,93 & 0,02 & 186 & 0.11 & 90 & 22 & 14,2 & 3,0 & 11,9 & 1,6 & 2,0 & 1,0 & 3,3 & 9,2 & 95 & 515 & 34 \\
\hline$A_{2}$ & 0.01 & 4,1 & 0.02 & $\$ 63$ & 0.04 & 106 & 21 & 18.2 & 0,6 & 2,5 & $1, t$ & 1.1 & 1,3 & 7.4 & 9.3 & 69 & 183 & 37 \\
\hline$A_{3}$ & 0,29 & 7,7 & 0.2 & 167 & 2.9 & 126 & 22 & 36.7 & 1,5 & 7,6 & 2,6 & 2,1 & 1,7 & 7.7 & 10,4 & 68 & 620 & 87 \\
\hline$A_{6}$ & 0.64 & 14,5 & 0.46 & 142 & 2.9 & 125 & 22 & 49,2 & 2,0 & 10,7 & 2.7 & 2.5 & 2.7 & 7.4 & 11.5 & 87 & 690 & IIs \\
\hline As & 0,39 & 14.6 & 0,18 & 132 & 2.7 & 128 & 21 & 14,7 & 1,1 & 3,1 & 6,2 & 6,1 & 2,7 & 7,5 & 11,3 & $\cos$ & 676 & 119 \\
\hline 16 & 1,0 & 13.2 & 10,0 & 136 & 7.0 & 100 & 17 & 46,9 & 9.2 & 4,8 & 4,8 & 4,6 & 5,4 & 2.4 & 16,0 & $9 a$ & 645 & 68 \\
\hline hJ & 1.0 & 10.1 & 2,4 & 153 & 5.4 & 116 & 17 & 48.1 & 11,8 & 9,5 & 3,4 & 3,4 & 5.6 & 7.6 & 13,1 & 89 & 680 & 112 \\
\hline$A_{B}$ & 1.16 & 18.0 & 1,0 & 146 & 6,9 & 120 & 19 & 44,7 & 18,0 & 22,7 & 2,8 & 2,1 & 5,9 & 7,3 & 15,7 & 107 & 618 & t 28 \\
\hline A & 0,06 & 19,0 & 1,1 & 140 & 5,7 & 117 & 10 & 46,2 & 23,3 & 9,6 & 3,0 & 3,0 & 5,6 & 7.4 & 13,9 & 102 & 720 & 112 \\
\hline$a_{1}$ & 0.06 & 12,3 & 0,02 & 157 & 0.05 & 926 & 21 & 35,8 & 2 & 3,7 & 1.4 & 1.6 & 2.2 & 7.7 & 10,4 & 91 & 631 & 74 \\
\hline B2 2 & 0,02 & 2,0 & 0.01 & 106 & 0.045 & 76 & is & 17.2 & 1.4 & 10,6 & 4,2 & 4,5 & 1,4 & 7,3 & 12,3 & 103 & 370 & 42 \\
\hline E & 0,03 & 2.4 & 0.01 & 127 & 0,23 & as & 12 & 26,9 & 2,9 & 7,4 & 2,4 & 2,6 & 1,3 & 9,1 & 12,8 & 103 & 428 & 29 \\
\hline $8_{6}$ & 0,18 & 12,6 & 0,05 & $|5|$ & 1.21 & 116 & 19 & 42.4 & 51.6 & 11,2 & 2,2 & 2,3 & 2,5 & 7,5 & 13,6 & 108 & 687 & 79 \\
\hline is & 0,03 & 7,2 & 0,11 & 156 & $0,0]$ & 129 & 12 & 42,3 & 12,3 & 7,4 & 1,3 & 1,4 & 2,4 & 7.3 & 11.9 & 117 & 647 & 82 \\
\hline$t_{6}$ & 1.2 & 11,5 & 2,7 & 127 & 4.4 & 168 & 22 & 48,5 & 13 & 28,6 & 3,8 & 9,5 & 7,2 & 0.1 & 13,8 & 91 & 762 & 271 \\
\hline 87 & $0,8 \mathrm{t}$ & 13,0 & 1,4 & 160 & 4,6 & 123 & 17 & 33 & 10,5 & 7,1 & 3,1 & 3,1 & 4.7 & 7,6 & 11,1 & 43 & 310 & $11 B$ \\
\hline 20 & 0.05 & 4,7 & 0.06 & 113 & 0.18 & 91 & 16 & 25.5 & 3,3 & 7,0 & 2,2 & 2,1 & 3,3 & 7,7 & 11,3 & 102 & 425 & 72 \\
\hline
\end{tabular}




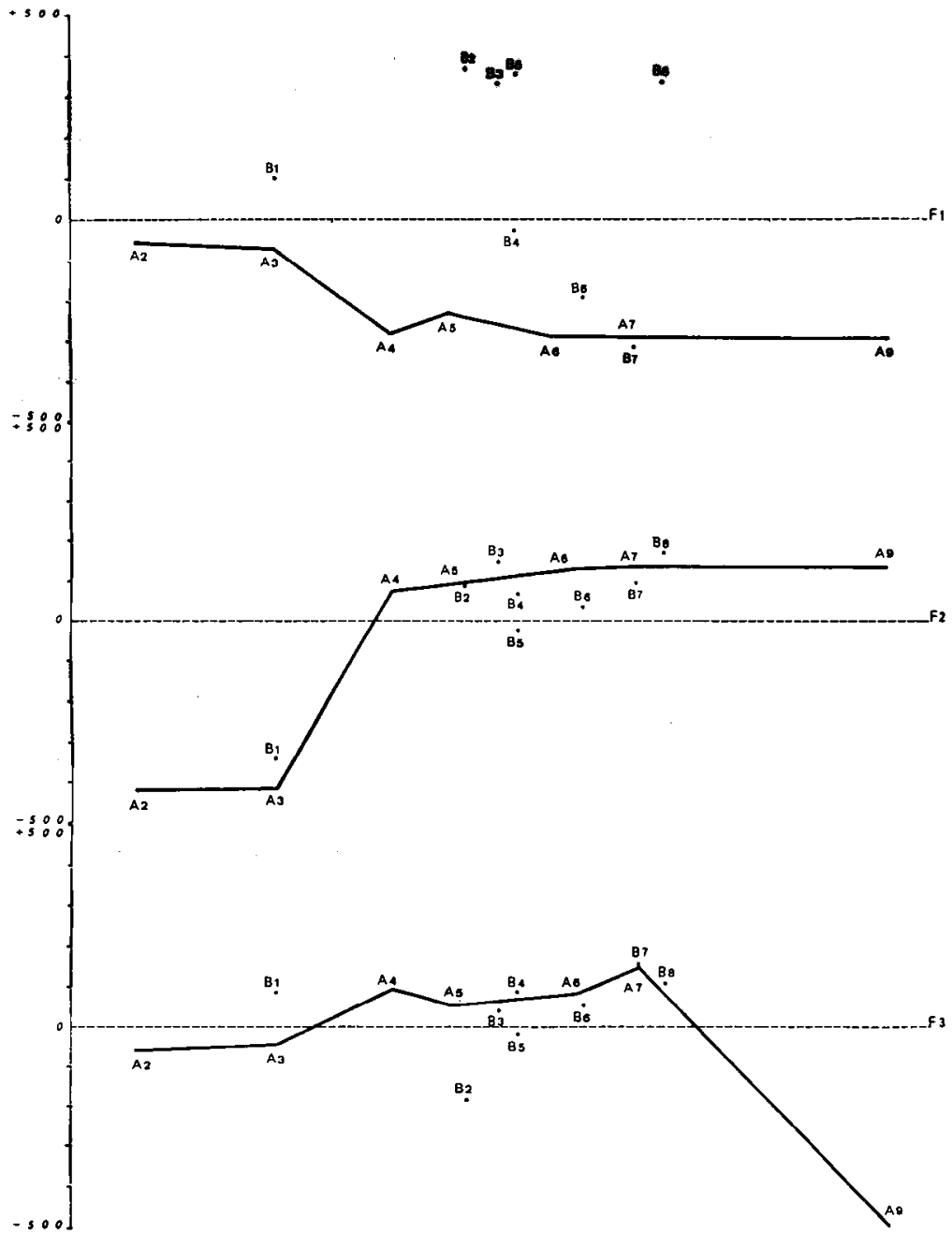

Fig. 2. Coordonnées des stations sur les axes I, II, III. 
Comparativement aux études citées, l'Arc apparaît comme un milieu particulièrement perturbé et désorganisé par les contaminants provenant des activités anthropiques implantées sur son bassin versant ; l'évolution spatio-temporelle naturelle de la rivière a disparu, ce qui n'est pas encore le cas du Buëch pourtant atteint par une importante pollution organique chronique alors que le fonctionnement du Lot est plus proche de celui de l'Arc. Les éléments allogènes jouent un rôle prépondérant dans le fonctionnement de cet hydrosystème méditerranéen et masquent son eutrophisation naturelle. Seuls quelques affluents exempts d'impacts permettent encore de percevoir quel pouvait être l'état antérieur de l'Arc avant qu'il ne soit soumis aux multiples perturbations, lot habituel des réseaux hydrographiques en région de forte densité humaine.

\section{Les peuplements de l'Arc}

La richesse des invertébrés benthiques du réseau de l' $\Lambda$ rc reflète une grande hétérogénéité des milieux prospectés. Ainsi les affluents qui sont soit des cours d'eau naturels, soit des cours d'eau très pollués, sont susceptibles d'abriter des biocénoses très diffèrentes. L'inventaire faunistique général réunit 216 taxons dont 35 espèces d'oligochètes. Cette diversité est comparable ou supérieure à celle des autres systèmes lotiques circumméditerranéens. On note ainsi 207 taxons dans la Moyenne Durance (Prévot 1984), 198 dans l'Argens du Var (Giudicelli et al. 1980), 186 dans la Restonica de Corse (Giudicelli 1968), 148 dans le Buëch des Hautes-Alpes (Vespini 1985), 130 dans la Basse Ardèche (Doledec 1985), 147 dans le Bas Verdon (Galvin 1989), 195 dans le Haut Sebou du Maroc (Dakki 1979) et 201 dans 2 cours d'eau du Mont Liban (Dia 1983).

Les nombreuses informations recueillies dans l'Arc ont été rassemblées et étudiées au moyen d'analyses multivariées.

La synthèse des données a été réalisée à partir d'une matrice de données taxons-stations de dimensions $151 \times 15$, traitée par une analyse factorielle des correspondances ( 15 stations ont été étudiées, 7 sur le cours axial, 8 sur les affluents (fig. 1) ; la station Al temporairement exondée et la station A8 difficilement accessible, $n$ 'ont pas été exploitées du point de vue faunistique.

Sur les 216 taxons initiaux, 151 seulement ont été retenus ; les 65 restants correspondant à 35 espèces
d'Oligochètes non dénombrées et 30 taxons représentés par un seul individu. A chaque couple taxonstation est associée la somme des individus récoltés au cours de 9 campagnes. Cet effectif correspond pour chaque station a $3,6 \mathrm{~m}^{2}$ de substrat échantillonné (soit $0,1 \mathrm{~m}^{2}$ de surface par surber, 4 prélèvements simultanés par station et 9 campagnes dans l'année). Les effectifs sont transformés en classes d'abondance de progression logarithmique de raison 0,6 .

\subsection{Caractéristiques et interprétation des axes factoriels}

Le cumul de l'information contenue dans les 3 premiers axes dépasse $54 \%$. L'interprétation de la signification de ces 3 axes devrait permettre d'extraire les caractéristiques fondamentales de la communauté animale benthique du réseau de l'Arc, et les influences qui déterminent son organisation.

\begin{tabular}{lccc}
\hline & Valeur propre & $\%$ d'inertie & Inertie cumulée \\
\hline Axe 1 & 0,072 & 27,7 & 27,7 \\
Axe 2 & 0,042 & 16,1 & 43,8 \\
Axe 3 & 0,027 & 10,3 & 54,1 \\
\hline
\end{tabular}

Ordination des stations sur l'axe factoriel (fig. 3) : deux lots de points-stations s'opposent sur l'axe I :

- les points de coordonnées positives représentent les affluents dont le chimisme est encore " naturel " dans le contexte régional ; ce sont les stations B2, B3, B5 et B8. A l'exception de B5, ce sont celles qui possèdent les contributions les plus élevées sur cet axe.

- sur la partie négative se situent les coordonnées de tous les points-stations du cours axial (A2 à $A 9$ ) et celles de 2 affluents où une perturbation $\mathrm{a}$ été décelée (B6 et $\mathrm{B} 7$ ).

- en position intermédiaire se situent les 2 affluents B1 et B4.

L'axe factoriel I traduit la distribution spatiale des stations d'étude en fonction de la réponse des communautés d'Invertébrés benthiques à la qualité de leur hydroclimat. Les stations regroupées par l'analyse présentent des affinités dans leurs peuplements dont la composition, la structure et la diversité taxonomique sont induites par un même contexte abiotique. On remarque, de plus, que l'ordonnancement sur l'axe I des stations du cours principal ne suit pas leur succession géographique 


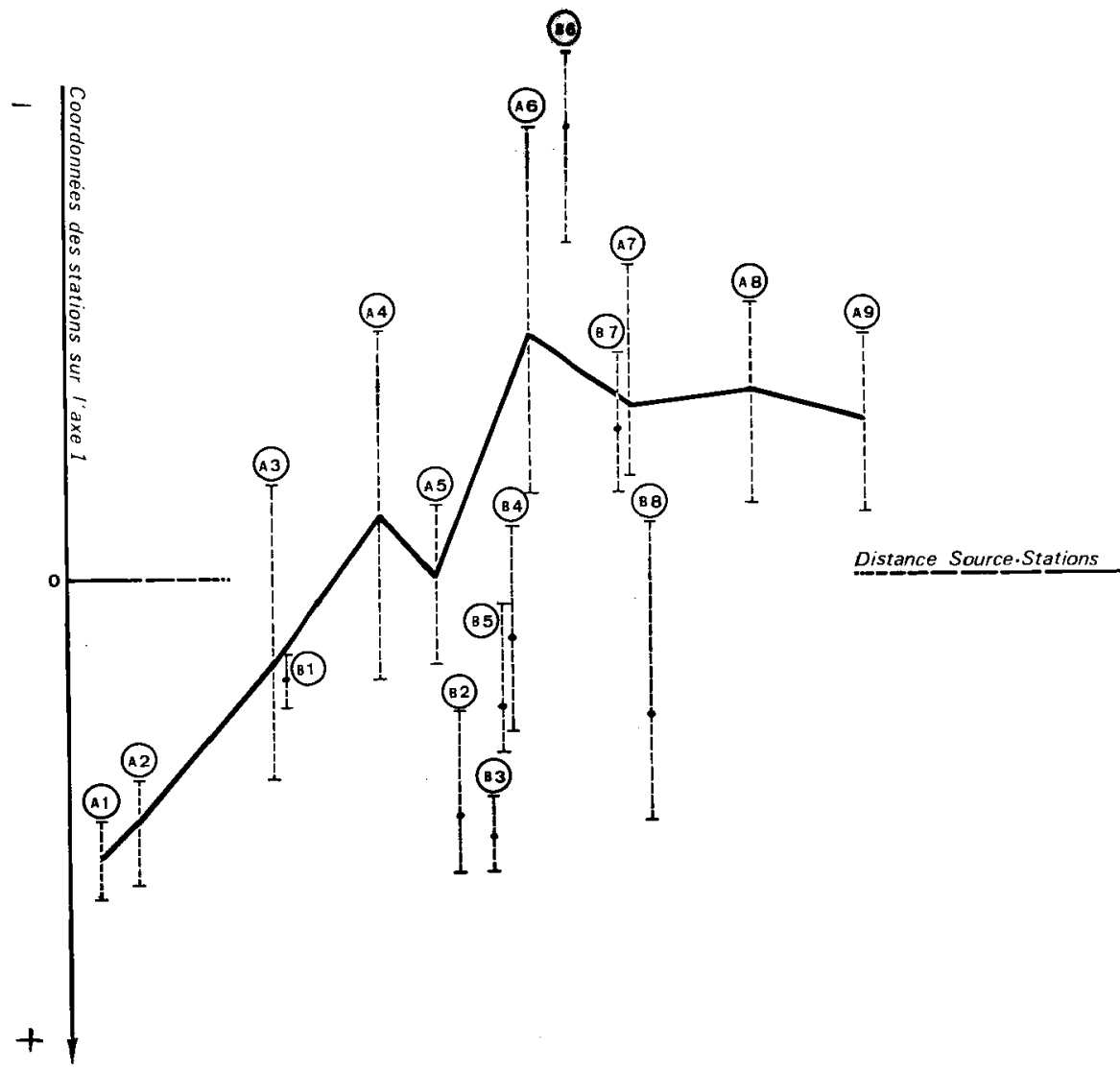

Fig. 3. Evolution de la moyenne annuelle des coordonnées stationelles de l'Arc et de ses afluents sur l'axe 1 de l'A.C.P. en fonction de leur distance à la source. 
naturelle. Cette disposition montre que la répartition de la faune benthique dans l'Arc est soumise à un facteur dominant : l'a hydrochimie ", non dépendant de l'évolution amont-aval telle qu'on la retrouve dans d'autres milieux lotiques plus ou moins perturbés : Verneaux (1973) dans le Doubs, Giudicelli et al. (1980) dans l'Argens.

Ici, l'étude faunistique confirme les conclusions émises au terme de l'analyse des descripteurs physico-chimiques du réseau de l'Arc, à savoir l'importance du phénomène d'eutrophication dans le fonctionnement de cet écosystème. Le peuplement d'invertébrés benthiques traduit l'impact des perturbations sur la totalité du cours principal de l'Arc ; les premiers rejets polluants ont lieu en A3. En effet, toutes les stations situées en aval de A3 restent groupées dans un nuage de points de coordonnées nettement négatives, malgré une situation hydrochimique proche de la " normale » en aval des rejets. L'autoépuration qui se réalise dans un contexte déjà nettement eutrophisé n'est pas révélée par les communautés d'invertébrés ; celles-ci se montrent ainsi de meilleurs indicateurs de pollution que les paramètres abiotiques. Ces observations sont en accord avec la remarque émise par Vespini (1985) vis-à-vis de l'évolution temporelle des peuplements benthiques du Buëch : « le benthos garde la marque de la pollution $"$.

Ordination des stations sur l'axe factoriel II (fig. 3) : l'axe II isole très nettement les peuplements des stations du haut réseau de l'Arc : A2, A3 et B1. Les paramètres hydrologiques jouent un rôle discriminant puisque ces 3 stations présentent un régime hydrique (quasi temporarité ou étiages sévères) très sélectif vis-à-vis des organismes benthiques. Ces 3 points ont des contributions très élevées sur le pôle négatif de l'axe II. Les stations de coordonnées positives ont un débit généralement important (stations du cours axial : A4 à A9 et des 4 affluents : B3, B6, $B 8$ ) ou qui n'atteint jamais un niveau critique, même en période d'étiage : B2, B4.

Ordination des stations sur l'axe factoriel IIl (fig. 3) : l'axe III isole la station A9.

\subsection{Analyse des espèces caractéristiques au niveau stationnel}

La projection des points espèces sur les cartes factorielles $\mathrm{I} / \mathrm{II}$ et $\mathrm{I} / \mathrm{III}$, conduit à mettre en évidence, à travers les changements de composition et de struc- ture, l'étroite dépendance qui lie la communauté benthique à son hydroclimat.

La figure 4 regroupe ces résultats faunistiques. Elle prend en compte les taxons qui ont les plus fortes contributions sur les axes I, II et III ainsi que certains taxons qui, du fait de leur valence écologique étroite, ont la valeur d'indicateurs de zones.

Trois groupes d'espèces ont été séparés dans le plan I/II par une classification hiérarchique ascendante réalisée à partir de leurs coordonnées sur les 3 premiers axes.

1) Un premier ensemble se trouve dans l'espace factoriel occupé par le groupement « affluents naturels » (B2, B3, B5, B8) mis en évidence dans le quadrant supérieur droit du plan I/II. Parmi ces espèces, certaines sont exclusives de ces sites ; ce sont les Ephémères Baetis muticus et Ephemera danica, le Plécoptère Leuctra geniculata, les Trichoptères Wormaldia occipitalis, Tinodes sp. et les Coléoptères Orectochilus villosus et Limnius intermedius. Cordulegaster annulatus, Onychogomphus uncatus, Sericostoma galeatum, Psychomyia pusilla, Esolus pygmaeus et Riolus cupreus, présentent leur abondance maximale dans ces stations. Enfin, quelques espèces peu abondantes, dont les contributions aux axes I et II sont faibles, sont exclusives de ces $\mathbf{4}$ stations : Perla marginata, Agapetus cravensis, Silo nigricornis.

Les espèces, abondantes et/ou exclusives qui constituent ce noyau, ont une signification écologique assez précise et caractérisent bien le type de biotope correspondant à ce premier groupement de stations.

Certaines sont considérées comme potamophiles :

- Giudicelli et al. (1980) signalent l'abondance de Leuctra geniculata et Limnius intermedius dans les stations aval de l'Argens ; ces 2 espèces sont qualifiées d'《 accessoires " dans la communauté benthique du métarhithral et de l'hyporhithral. Pour Verneaux (1973), Leuctra geniculata est une espèce structurale du cours inférieur de la Loue et appartient à l'hyporhithron du Doubs. Limnius volckmari dont la répartition dans l'Arc est, comme dans l'Argens, presque superposable à celle de $L$. intermedius est, par contre, caractéristique des secteurs supérieurs des réseaux.

- Rhyacophila dorsalis, dont la distribution dans le réseau de l'Arc est limitée aux affluents, est présente sur tout le cours de l'Argens dans 


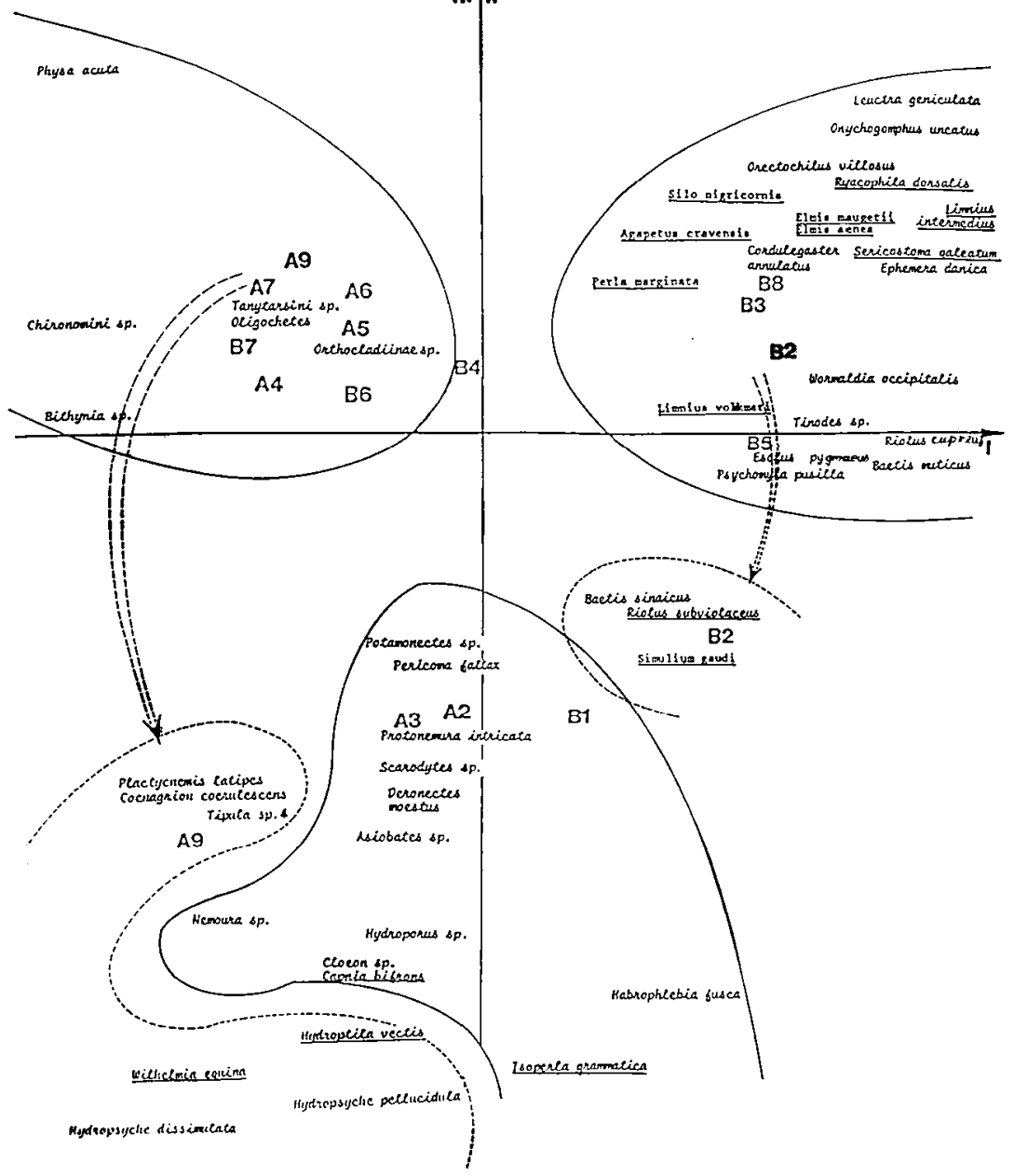

La figure visualise :

Fig. 4. Superposition des plans factoriels $\mathrm{I} / \mathrm{II}$ et $\mathrm{I} / \mathrm{III}$.

- les taxons les plus contributifs (en italique) aux axes factoriels : I, II (taxons regroupés par un trait plein) et III (taxons regroupés par un trait en pointillés).

- les taxons ayant valeur d'indicateurs de zones biocénotiques (en caractères droits, soulignés).

- les taxons très contributifs et indicateurs de zoncs biocénotiques (en italique, soulignes).

- la projection des points-stations dans le plan I/II et la translation des points A9 et B2 selon l'axe III. 
l'épipotamal duquel l'espèce est classée parmi les espèces compagnes (Giudicelli et al. 1980). Décamps $(1967,1968)$ signale $R$. dorsalis comme la plus potamophile des Rhyacophila du bassin de la Garonne. Dans le Doubs, elle marque le passage entre le rhithron et le potamon (Verneaux 1973). En fait, Moretti \& Mearelly (1981), dans une étude sur l'autoécologie de certains $R$ hyacophila d'Italie, ont montré que $R$. dorsalis a un très large profil écologique.

- Psychomyia pusilla, élective des affluents naturels de l'Arc, marque également une préférence pour les secteurs moyen et inférieur d'autres réseaux méditerranéens : Giudicelli (1968) la signale dans l'hyporhithron et l'épipotamon du réseau du Tavignano (Corse) et dans le cours inférieur de l'Argens (Giudicelli et al. 1980). Dans le Doubs (Verneaux 1973) et les réseaux des Pyrénées (Décamps 1967), ce Trichoptère est également classé dans un biocénotype de transition entre le rhithron et le potamon.

Les affluents non perturbés hébergent, ainsi, un ensemble d'espèces dont certaines réalisent habituellement leur optimum écologique dans le cours inférieur des rivières. Elles ont été éliminées du potamon de l'Arc par les importantes perturbations qui s'y exercent mais elles subsistent dans les affluents qui constituent la limite de leur aire de distribution zonale.

Les autres espèces de ce groupement ont, par contre, pour biotope préférentiel la zone supérieure des réseaux :

- Les Trichoptères Sericostoma galeatum et Agapetus cravensis sont des espèces méridionales. En dehors de la communauté du métarhithral et de l'hyporhithral de l'Argens dont elle constitue la seule espèce exclusive, $A$. cravensis n'avait été trouvée que dans des sources de Provence. Elle est présente dans 2 affluents du réseau de l'Arc : B3 et B8. Sericostoma galeatum est une espèce assez eurytope mais préférente du métarhithral et de l'hyporhithral de l'Argens (Giudicelli et al. 1980) ; elle a été signalée également dans d'autres cours d'eau du Sud-Est de la France, la Moyenne Durance (Prévot 1984) et dans le Nord de l'Italie (Moretti \& Cianficconi 1977).

- Silo nigricornis, exclusive de B3 et de B8, peuple en France les sources, ruisseaux et rivières (Grenier et al. 1969).
C'est une espèce prêférente du crénal et des secteurs initiaux du rhithral dans l'Argens (Giudicelli et al. 1980), classification qui s'accorde avec celle de Verneaux (1973) dans le Doubs (épirhithron).

- Seul, le peuplement du Grand Torrent (B8) renferme le Plécoptère Perla marginata. Cette espèce, à large répartition européenne et maghrébine, est inféodée au rhithral sensu lato.

Le peuplement benthique du groupe de stations B2, B3, B5, B8, du fait de la signification zonale de la majorité des espèces qui le caractérise, se rattache globalement au biocénotype du rhithron. Il comprend également quelques espèces potamophiles (de l'épipotamon) pour lesquelles ces affluents peu perturbés constituent, à l'échelle du réseau de l'Arc, des habitats refuges.

Ce groupement d'espèces se rapporte aux seuls affluents naturels; aucun groupement équivalent n'a pu être mis en évidence dans le cours axial de l'Arc. Il semble cependant, qu'une telle association pourrait caractériser le cours moyen de l'Arc en l'absence de toute perturbation.

On peut admettre que le peuplement benthique de ces affluents représente la « mémoire » du peuplement originel de l'Arc et qu'en conséquence, il constitue un réservoir d'espèces caractéristiques du rhithron, zone disparue dans le cours principal de l'Arc en raison d'une trop forte pression anthropique.

2) Les peuplements benthiques des stations du haut réseau ( $B 1, A 2, A 3)$, situés au contact des 2 quadrants inférieurs du plan $\mathrm{I} / \mathrm{II}$, sont composés de nombreuses espèces exclusives du secteur amont de l'Arc : Potamonectes sp., Scarodytes sp., Asiobates sp., Hydroporus sp., Deronectes moestus, Protonemura intricata, Capnia bifrons, Pericoma fallax et Cloeon sp.. Isoperla grammatica, Nemoura $s p$. et Habrophlebia fusca réalisent leur optimum écologique dans ces 3 stations à régime quasitemporaire ou à faible débit.

Le groupe des Plécoptères est surtout représenté dans ces milieux par Isoperla grammatica (espèce univoltine) ainsi que par Nemoura cinerea et Capnia bifrons, espèces présentant des arrêts de développement pendant certaines périodes du cycle hydrologique du ruisseau. Isoperla grammatica, espèce eurytope dans toute l'Europe, a été récoltée dans les ruisseaux temporaires de Provence par 
Bouraima (1974) et Bouzidi (1983). Capnia bifrons et Nemoura cinerea sont bien adaptées à l'habitat temporaire; Légier (1979) les a récoltées dans des ruisseaux temporaires de Provence calcaire. Les Ephéméroptères Habrophlebia fusca et Cloeon sp. sont particulièrement abondantes dans ces trois stations. Les Coléoptères Hydrocanthares sont adaptés à la colonisation des milieux temporaires grâce à leur capacité de vol ; ils vivent dans les eaux stagnantes ou dans les eaux claires à faible courant et migrent au moment de l'assèchement des biotopes aquatiques. Le peuplement du haut réseau de l'Arc renferme de nombreuses espèces de Coléoptères dont la présence coïncide avec la phase lénitique du cycle hydrologique. L'abondance des Plécoptères, par contre, est élevée au cours de la phase lotique.

Les peuplements benthiques des 3 stations du cours supérieur de l'Arc sont caractérisés par un groupement d'espèces lénitophiles et adaptées aux milieux temporaires. Ils colonisent ainsi des biotopes très particuliers, limités dans le réseau de l'Arc aux premiers kilomètres du cours principal et à un affluent de faible débit, ce qui explique leur position isolée à l'extrémité négative de l'axe II de l'A.F.C.

3) Quelques taxons saprobiontes, très abondants, contribuent à la formation du noyau de stations perturbés (A4 à A7, B6, B7) situées dans le quadrant supérieur gauche de la figure 4.

Les Diptères Chironomidae (Chironomini, Tanytarsini et Orthocladiinae) bien connus pour leur polluo-résistance et les Oligochètes, dont les fortes densités sont indicatrices de pollution organique (Giani 1984), dominent dans les peuplements de A6, A7, B6 et B7. Les Mollusques Physa acuta et Bithynia sp. colonisent surtout les macrophytes, très développés en A4 et A5. Les Coléoptères Elmidae sont bien diversifiés dans le réseau de l'Arc : 12 espèces sont recensées, comme dans la rivière Argens (Giudicelli et al. 1980). Cependant, leurs représentants sont rares dans les peuplements du noyau de stations perturbées et même complètement absents en A4, A6 et B7. Leur répartition longitudinale dans les Pyrénées (Berthélémy 1966) ainsi que dans l'Argens, est caractérisée par l'augmentation du nombre de genres et d'espèces de l'amont vers l'aval. Dans la majeure partie du cours principal de l'Arc, l'absence
d'Elmidae résulte d'une forte pollution organique Celle-ci, par contre, est favorable à la prolifération des espèces saprobiontes caractéristiques du noyau de stations perturbées isolées sur la partie négative de l'axe I.

Les stations A9 et B2 occupent dans le plan I/III de l'analyse une position particulière, éloignée des noyaux des stations affines auxquelles elles appartiennent dans le plan I/II. Les espèces qui déterminent cette translation ont des contributions élevées sur l'axe III.

- En ce qui concerne la station A9: Hydropsyche dissimulata, Platycnemis latipes, Coenagrion coerulescens et Wilhelmia equina sont exclusives. $H y d r o p s y c h e$ pellucidula et Hydroptila vectis y sont très abondantes. $H$. pellucidula, véritablement eurytope dans l'Argens, présente dans l'Arc son optimum écologique en A9. W. equina, caractéristique du métarhithron et de l'hyporhithron (Verneaux 1973), est exclusive, comme $H$. dissimulata, de cette station. $\boldsymbol{H}$. vectis est classée par Verneaux dans l'épipotamon ; hormis quelques individus récoltés en $\mathbf{B} 3$, cette espèce n'est abondante qu'en A9. Platycnemis latipes est une espèce de fleuves et rivières à courant lent. Cet ensemble d'espèces distingue ainsi très nettement A9 des autres stations du cours axial de l'Arc. Il caractérise un peuplement mieux diversifié, inféodé au potamon méditerranéen et témoignant d'une légère restauration de l'hydroclimat.

- Dans la station B2, Simulium gaudi est une espèce caractéristique des zones de collines et des ruisseaux pétrifiants à formation encroûtante (Dorier 1963) ; nous l'avons rencontrée dans quelques rares cours d'eau des Alpes Maritimes (Le Loup) et du Var (la Siagnole, le Caramy et l'Argens). Les eaux calcaires et le substrat encroûté de B2 constituent son biotope préférentiel dans le réseau de l'Arc. Riolus subviolaceus, dont l'abondance dans cette station est également liée à la nature encroûtée de substrat (Berthélémy 1966), est une espèce eurytope mais souvent élective du cours supérieur des réseaux. Ainsi, dans l'Argens, Giudicelli et al. (1980) la signalent dans les 5 stations amont du réseau. Riolus subviolaceus et Baetis sinaicus dont les contributions sur l'axe III de l'A.F.C. sont élevées, constituent avec $S$. gaudi un groupement d'espèces associé à cette seule station. 


\section{Conclusion}

L'analyse des peuplements benthiques du réseau de l'Arc, par l'utilisation de l'A.F.C., permet de réaliser une synthèse des données faunistiques malgré l'hétérogénéité de cet hydrosystème et le nombre important de sites choisis. Cette A.F.C. sépare les stations en 3 groupes représentant chacun une certaine qualité de l'environnement déjà mise en évidence par l'A.C.P. concernant la physico-chimie des eaux de ces mêmes stations.

Les stations du cours supérieur : A2, A3, B1, sont caractérisées par un groupement d'espèces lénitophiles et adaptées aux milieux temporaires. Sur le cours principal, les stations perturbèes $\mathrm{A} 4$ à $\mathrm{A} 7$ sont marquées par la prolifération des espèces saprobiontes (ainsi que les affluents B6 et B7) alors que le peuplement mieux diversifié de $\mathrm{A} 9$, inféodé au potamon Méditerranéen, témoigne d'un début d'autoépuration.

Les affluents permanents non perturbés : B2, B3, B5, B8, hébergent des peuplements benthiques se rattachant globalement à l'hyporhithron auquel se joignent quelques espèces de l'épipotamon.

L'absence de communautés naturelles dans le secteur moyen de l'Arc d'une part, la présence de communautés hyporhithrales et épipotamales dans les affluents non perturbés d'autre part, laissent à penser que le peuplement benthique de ces affluents représente à la fois une «mémoire » du peuplement originel du cours d'eau axial et donc un réservoir d'espèces susceptibles de le recoloniser dans le cas d'une réduction de la pression anthropique actuelle.

\section{Travaux cités}

Angelier (E.), Bordes (J.M.), Lucchetta (J.C.) \& Rochard (M.). 1978. - Analyse statistique des paramètres physicochimiques de la rivière Lot. Annis Limnol. 14 (1-2) : 39-57.

Berthèlémy (C). 1966. - Recherches écologiques et biogeographiques sur les Plécoptères et Coléoptères d'eau courante (Hydraena et Elminthidae des Pyrénées. Annls Limnol. 2 (2) : 227.458 .

Bouraima (I.). 1974. - Recherches d'écologie quantitative sur la zoocénose de deux ruisseaux temporaires en BasseProvence. Thèse 3ème cycle Aix-Marseille IJI : 203 p.

Bouzidi (A.). 1983. - Cours d'eau temporaires du massif des Maures. Hydrologie, Hydrochimie, organisation et évolution de leur peuplement. Thèse 3ème cycle, Université Aix. Marseille [1] : $190 \mathrm{p}$.

Dakki (M.). 1979. - Recherches hydrobiologiques sur un cours d'eau du Moyen-Atlas (Maroc). Thèse 3ème cycle, Université Aix-Marseille III : $126 \mathrm{p}$

Décamps (H.), 1967. - Recherches écologiques sur les Trichoptères des Pyrénèes. Ann's Limnol 3 (1): 101-176.

Décamps (H.). 1968. - Vicariance écologique chez les Trichoptères des Pyrénées. Annis Limnol. 4 (1) : 1 -50.
Dia (A.). 1978. - Etude écologique et essai de zonation d'une rivière côtière méditerranéenne l'Argens (Var). Thèse 3ème cycle, Aix-Marseillc III : 155 p.

Dia (A.). 1983. - Recherches sur l'écologie et la biogéographie des cours d'eau du Liban méridional. Thèse de doctorat d'état, Université Aix-Marseille III : 302 p.

Doledec (S.). 1986. - Les peuplements de macro-invertébrés du cours inférieur de l'Ardèche. Dynamique spatio-temporelle. Thèse doctorat, Université Claude Bernard Lyon I : 246 p.

Dorier (A.). 1963. - Documents pour servir à la connaissance des Simuliidae du Sud-Est de la France. Trav. Lab. Hydr. Pisc. Grenoble 54.55: 7-79.

Duvignaud (P.). 1974. - La synthèse écologique. Populations, communautés, écosystèmes, biosphères, noosphères. Doin édit. Paris : $296 \mathrm{p}$.

Galvin-Chabrière (N.). 1982. - Evolution d'une charge polluante dans un cours d'eau : l'Argens (Var). Rôle d'un bief d'eau calme dans le fonctionnement du système. Thèse Jème cycle, Aix-Marseille III, 179 p.

Galvin (Y.). 1989. - Hydrobiologie d'une rivière aménagée : le Moyen-Verdon (Alpes de Haute Provence). Hydrochimie et Macro-invertébrés benthiques d'un tronçon à débit régulé. Impacts de l'aménagement sur l'hydrosystème. Thèse doctorat, Université d'Aix-Marseille III : $190 \mathrm{p}$.

Giani (N.). 1984. - Le Riou Mort, affluent du Lot pollué par métaux lourds. IV. Etude des oligochètes. Annis Limnol. 20 (3) : 167-181.

Giudicelli (J.). 1968. - Recherches sur le peuplement, l'écologic et la biogéographie d'un réseau hydrographique de la Corse centrale. Thèse de doctorat d'état, Université Aix-Marseille $I: 437 \mathrm{p}$.

Giudicelli (J.). Dia (A.) \& Légier (P.). 1980. - Etude hydrobiologique d'une rivière de région méditerranéenne : I'Argens (Var) : peuplements invertébrés et distribution longitudinale. Bijdr Dierkd, Ro: 303-341.

Grenier (S.), Décamps (H.) \& Giudicelli (J.). 1969. - Les larves de Goeridae (Trichoptera) de la faune de France. Taxonomie et Ecologie. Annis Limnol. 5 : 129-161.

Lavandier (P.) \& Mur (C.). 1974. - Ecologie d'un torrent pyrénéen de haute mongagne. I1. Caractéristiques chimiques. Annis Limnol. 10 (3) : 275-309.

Légier (P.). 1979. - Ecologie des ruisseaux temporaires de Provence et les informations qu'elle apporte sur la naissance, la maturation et la structure d'un écosystème d'eau courante. Thèse de doctorat d'état, Aix-Marseille III : 320 p.

Moretti (G.P.) \& Mearelly (M.). 1981. - Ecological profiles in three Rhyacophila species. Proc. of the 3rd Int. Symp. on Trichoptera, Junk, The Hague : 7-30.

Prévot (R.). 1984. - Hydrobiologie de la Moyenne Durance. Étude des différents milieux et de leurs peuplements de macroinvertébrés. Thèse 3ème cycle, Aix-Marseille III : $179 \mathrm{p}$.

Verneaux (J.). 1973. - Cours d'eau de Franche-Comté (Massif du Jura). Recherches écologiques sur le réseau hydrographique du Doubs. Essai de Biotypologie. Thèse de doctorat d'état, Univ. Besançon : $257 \mathrm{p}$

Vespini (F.). 1985. - Contribution à l'étude hydrobiologique du Buëch, rivière non aménagée de Haute Provence. Suivi d'une charge organique faible et mesure de ses effets sur les composantes physiques, chimiques et biologiques de l'écosystème. Thèse 3ème cycle, Aix-Marseille I : $148 \mathrm{p}$. 